\title{
Nanoparticles in Drug Delivery and Cancer Therapy: The Giant Rats Tail
}

\author{
Vinod Prabhu, Siddik Uzzaman, Viswanathan Mariammal Berlin Grace, \\ Chandrasekharan Guruvayoorappan*
}

${ }^{1}$ Department of Biotechnology, Karunya University, Karunya Nagar, Coimbatore, Tamil Nadu, India.

Email: "gurukarunya@gmail.com

Received January $17^{\text {th }}, 2011$; revised May $26^{\text {th }}, 2011$; accepted June $13^{\text {th }}, 2011$.

\begin{abstract}
Nanotechnology has the potential to offer solutions to these current obstacles in cancer therapies, because of its unique size and large surface-to-volume ratios. Nanoparticles may have properties of self-assembly, stability, specificity, drug encapsulation and biocompatibility as a result of their material composition. Nanoscale devices have impacted cancer biology at three levels: early detection, tumour imaging using radiocontrast nanoparticles or quantum dots; and drug delivery using nanovectors and hybrid nanoparticles. Other role of nanotechnology, in management of various diseases and also in drug resistance in leukemia by blocking drug efflux from cancer cells and induce efficient delivery of si RNA into lymphocytes to block apoptosis in sepsis and targeting tumors also. Nanocrystals labeling with immune cells can act as a platform technology for nanoimmunotherapy. This review addresses the advancement of nanoparticles in drug delivery and in cancer therapy.
\end{abstract}

Keywords: Nanoparticles, Cancer Therapy, Drug Delivery, Drug Targeting, Quantum Dots

\section{Introduction}

Nanotechnology deals with the design, production and characterization on ultra small particles which is extended to broad area in pharmaceutical, medical, chemical and engineering application due to its unique properties [1]. The development of technology occurs at the atomic, molecular or macromolecular range of approximately $1 \mathrm{~nm}$ - 100 nanometers $(\mathrm{nm})$ to create and use structures that have novel properties [2]. Nanoparticles (NPs) are defined as a small object that behaves as a whole unit in term of its transport and properties. They can be further classified according to the size and diameter. Fine particles have the range of 100 to $2500 \mathrm{~nm}$ or ultrafine particles having the size of 1 to $100 \mathrm{~nm}$ [3]. Nanoclusters have one dimension between 1 and $10 \mathrm{~nm}$ and narrow size distribution and nanopowders which are agglomerates of ultrafine particles [4]. NPs research is currently an area of passionate scientific interest due to its wide variety of potential application in therapeutic and biomedical interest. The field of nanotechnology holds the promise of significant improvements in the health and well being of patients as well in manufacturing technologies [5]. Specialized nanotechnological approaches like dendrimers, quantum dots, monoclonal antibodies and intergrins which are extensively researched for diagnostic and targeted delivery of therapeutic agents [6]. Nanorobotics centers on self sufficient machines of some functionally operating at the nanoscale. These are hopes for applying nanorobots in medicine [7-9]. The advance of contemporary materials and methodologies have to be manifested with some patients granted about new nano devices which will help in establishing NPs with the use of embedded nanobioelectronics concept in future $[10,11]$. Nanomedicine access to drug delivery on development of nanoscale molecules which can improve drug bioavailability $[12,13]$. It was disclosed that this can potentially be achieved by molecules targeting by nanoengineering devices. This new method will be effective in treating a variety of illness such as neurological disorders, diabetes, osteoporosis, Alzheimer's, Parkinson's, amyotrophic lateral sclerosis, multiple sclerosis, HIV-1 associated neuro cognitive disorders, cardio vascular disorders, tuberculosis and cancer [14-22]. NPs such as lipid or polymer can be designed to improve the pharmacological and therapeutical properties of drugs [21]. Cells take up these NPs because of their size and also the ability of the drug to get into the cell cytoplasm through cell membrane. NPs have a very high surface area to volume ratio and it allows many functional group 
to be attached to a NPs which can bind to certain tumor cells [22]. The smaller size of the NPs facilitates them to accumulate in tumor micro environment thus facilitating newer therapeutic strategies which may replace radiation and chemotherapy.

Recent research has developed a number of NPs such as metals, semiconductor and polymeric particles used in imaging probes and delivery vehicles [23-25]. The use of NPs based drug delivery systems such as polyethleneimine liposomes (PEI), silica NPs, micelles and chitosan have effective role in drug delivery with reduced drug side effects [26,27]. Recent technology have developed many multifunctional NPs for targeting, imaging, drug delivery, sensing of anticancer agents and small interference RNA (si RNA) delivery [28,29]. Nanotechnology promises construction of artificial cells, enzymes and genes that helps in the replacement therapy of many disorders which are due to deficiency of enzymes, mutation of genes or repair in the synthesis of protein [30]. In this review, we discuss the recent emerging trends of NPs in drug delivery system and in cancer therapy.

\section{Multifunctional Nanoparticles}

Liposome comprises lipid bilayer membrane surrounding an aqueous interior and it can be used as nanoparticles that have similarities with biological membrane that improves the efficacy and safety of drugs [31]. Liposomes are classified into three categories such as small unilamellar vesicles, large unilamellar vesicles and multilamellar vesicles on the basis of their size and lamellarity. The active compound can be located either in the aqueous spaces, if it is water-soluble, or in the lipid membrane, if it is lipid-soluble. The new generation of liposome called 'stealth liposomes' have the ability to evade the interception by the immune system, and have longer half-life [32]. The emulsions comprise of oil in water-type mixtures that are stabilized with surfactants to maintain size and shape. The lipophilic material can be dissolved in water organic solvent that is emulsified in an aqueous phase. Like liposomes, emulsions have been used for improving the efficacy and safety of diverse compounds [33]. Polymers such as polysaccharide chitosan NPs have been used for some time as drug delivery systems [34]. Water-soluble polymer hybrid constructs polymer-protein conjugation that reduces immunogenicity, prolongs plasma half-life and enhances protein stability. Polymer-drug conjugation promotes tumor targeting through the enhanced permeability and retention effect and at the cellular level following endocytic capture, allows lysosomotropic drug delivery [35]. Ceramic NPs are inorganic systems with porous characteristics that have emerged as drug vehicles [36]. These vehicles are biocompatible ceramic NPs such as silica, titania and alumina that can be used in cancer therapy. Gold shell NPs and other metal-based agents can serve as novel category of spherical NPs consisting of a dielectric core covered by a thin metallic shell which is typically gold. These particles possess highly favorable optical and chemical properties for biomedical imaging and therapeutic applications [37]. Carbon nanomaterials include fullerenes and nanotubes. Fullerenes are novel carbon allotropes with a polygonal structure made up exclusively by 60 carbon atoms. These NPs are characterized by having numerous points of attachment whose surfaces also can be functionalized for tissue binding [38]. Nanotubes have been one of the most extensively used types of NPs because of their high electrical conductivity and excellent strength. Carbon nanotubes can be structurally visualized as a single sheet of graphite rolled to form a seamless cylinder. There are two classes of carbon nanotubes that are single-walled (SWCNT) and multi-walled (MWCNT). MWCNT are larger and consist of many single-walled tubes stacked one inside the other compared to SWCNT. Functionalized carbon nanotubes are emerging as novel components in nanoformulations for the delivery of therapeutic molecules [39]. Quantum dots are NPs made of semiconductor materials with fluorescent properties which are mostly used in biological applications and quantum dots must be covered with other materials allowing dispersion and preventing leaking of the toxic heavy metals [40].

\subsection{Synthesis of Nanoparticles}

Size of the nanoparticle is very important for efficient drug delivery. Generally, $10 \mathrm{~nm}-100 \mathrm{~nm}$ is considered as the optimal size for nanoparticle drug carriers. If the particle size is less than $10 \mathrm{~nm}$, the NPs will be quickly eliminated by renal clearance (threshold $<6 \mathrm{~nm}$ ) and at sizes greater than $100 \mathrm{~nm}$, will have chances to be captured by the reticuloendothelial system (RES) [41]. Surface coating is essential for the stability and circulation time of NPs delivery system. For example a sodium citrate-stabilized gold particle aggregates in phosphatebuffered saline (PBS) within several minutes but once coated with thiol-terminated polyethylene glycol (PEG) polymer provides stability not only in PBS but also under low or high pH conditions [42]. Neutral charged NPs exhibits longer circulation time and reduce the chance of nanoparticle capture by the immune system.

\subsection{Gold Nanoparticles}

Nanoparticles synthesis and the study of their size and its properties are fundamental importance in the advancement of recent research [43]. It is exposed that optical, electronic, magnetic and catalytic properties of metal NPs depends on their size, shape and chemical sur- 
roundings. In NP synthesis it is important to control not only particles size but also the particle shape and morphology. Colloidal gold also known as nanogold is a suspension or colloid of sub-micrometer sizes particles of gold in a fluid-usually water [44]. Gold NPs can be produced in liquid chemical method by reduction of chloroauric acid $\left(\mathrm{HAuCl}_{4}\right)$. After dissolving $\mathrm{HAuCl} 4$ the solution is then rapidly stirred followed by the addition of reducing agents. This enhanced the production of $\mathrm{Au}^{3+}$ ions which gets reduced to neutral gold atoms. More and more of these gold atoms from the solution turns to precipitate in the form of sub nanometer particles. Vigorously stirring of this solution results in the production of particles of uniform size [44].

\subsection{Silver Nanoparticles}

The uniform silver NPs can be obtained by the reduction of silver ions by ethanol at $800^{\circ} \mathrm{C}$ to $1000^{\circ} \mathrm{C}$ under atmospheric condition [45]. In this synthesis process $20 \mathrm{ml}$ of aqueous solution containing silver nitrate $(0.5 \mathrm{~g}$ of $\left.\mathrm{AgNO}_{3}\right)$ can be treated with sodium linoleate $\left(\mathrm{C}_{18} \mathrm{H}_{32} \mathrm{O}_{2}\right)$ (1.5 g) in tubes under continuous agitation. The aqueous solution containing silver ions and sodium linoleate can be further treated with a mixture of linoleic acid and ethanol resulting in the formation of an ethanol solution phase containing silver ions. The ethanol in the liquid and solution phase reduces the silver ions into silver NPs. Linoleic acid will be absorbed on the surface of the silver NPs with alkyl chains on the outer side in a circular shape. Wang [46] demonstrated that on changing the concentration of the electrolyte a reddish brown color developed on addition of linoleic acid their by indicating $100 \%$ conversion of silver ions into silver NPs.

\subsection{Copper Nanoparticles}

A novel method for the preparation of copper NPs is by reducing the copper sulphate $\left(\mathrm{CuSO}_{4}\right)$ with hydrazine in ethylene glycol under microwave irradiation. The heating method and reaction temperature on the particle size and composition of powder have been investigated by X-ray diffractometry (XRD) and transmission electron microscopy (TEM). Well-dispersed copper nanopowder with a diameter of $15 \mathrm{~nm}$ can be obtained in the absence of a protective polymer [47]. In order to obtain pure-phase copper NPs using water, the reaction time of $8 \mathrm{hr}$ is essential. Owing to the reduction property of ethylene glycol, the reaction rate using ethylene glycol is higher. In addition, the amount of reduction agent can be reduced largely. Polyvinylpyrrolidone (PVP) plays greater role on the size of copper particles, and increase in the (PVP) concentration that attributes to the smaller dimension particles. The mean diameter is about $4 \mathrm{~nm}$ when the concentration of PVP is $0.5 \mathrm{mmol} / \mathrm{L}$. PVP acts as the polymeric capping agents in the reaction preventing the agglomeration of the copper NPs. When water is the reaction medium, the $\mathrm{Cu}^{2+}$ complex is reduced to $\mathrm{Cu}^{+}$ complex and further reduction of $\mathrm{Cu}^{+}$will form the pure copper NPs [48].

\subsection{Microencapsulation of Nanoparticles}

There are various methods available for the microencapsulation but most useable methods are as follow: The particles are tumbled in a pan or other device while the coating material is applied slowly. Air-suspension coating of particles by solutions gives better control and flexibility. The particles are coated while suspended in an upward-moving air stream. They are supported by a perforated plate having different patterns of holes inside and outside a cylindrical insert. Just sufficient air is permitted to rise through the outer annular space to fluidize the settling particles. Most of the rising air (usually heated) flows inside the cylinder, causing the particles to rise rapidly. At the top, as the air stream diverges and slows, they settle back onto the outer bed and move downward to repeat the cycle. The particles pass through the inner cylinder many times in a few minutes. Spray drying serves as a microencapsulation technique when an active material is dissolved or suspended in a melt or polymersolution and becomes trapped in the dried particle. The main advantage is the ability to handle labile materials because of the short contact time in the dryer. In addition, the operation is economical. In modern spray dryers the viscosity of the solutions to be sprayed can be as high as $300 \mathrm{mPa} \cdot \mathrm{s}$. In chemical method the two reactants in a polycondensation meet at an interface and react rapidly. The basis of this method is the classical SchottenBaumann reaction between an acid chloride and a compound containing an active hydrogen atom, such as an amine or alcohol, polyesters, polyurea, polyurethane. Under the right conditions, thin flexible walls form rapidly at the interface. A solution of the pesticide and a diacid chloride are emulsified in water and an aqueous solution containing an amine and a polyfunctional isocyanate is added. To neutralize the acid formed during the reaction base may be added. Condensed polymer walls form instantaneously at the interface of the emulsion droplets. In a number of processes, a core material is imbedded in a polymeric matrix during formation of the particles. A simple method of this type is spray-drying, in which the particle is formed by evaporation of the solvent from the matrix material. However, the solidification of the matrix also can be caused by a chemical change [49].

\subsection{Advancement of Nanoparticle Based Drug Delivery System}

The important technological advantages of NPs used on 
drug carrier are high stability, high carrier capacity, feasibility of incorporation of both hydrophilic, hydrophobic substances and feasibility of variable routes of administration including oral application and inhalation [50]. The NPs can also be designed to allow controlled sustained drug release from the matrix. These properties will enhance to improve the drug bioavailability and reduces of dosing frequency and prevent non adherence to prescribed therapy. Micelles so called core shell structure in which the core of the micelles which is either the hydrophobic part or the ionic part of the NPs can contains small or bigger therapeutic drug [51]. The novel intracellular $\mathrm{pH}$ sensitive polymeric micelles drug carrier which control the systemic and sub cellular distribution of pharmacologically active drug. The micelles can be prepared from self assembling amphiphilic block copolymers, poly (ethylene glycol), poly (aspirate hydrazone adriamycin) to which the adriamycin (anticancer drug) is conjugated to the hydrophobic segments by acid sensitive hydrazone linkers. Therefore micelles can preserve drug under $\mathrm{pH} 7.4$ and can release them by sensing the intracellular $\mathrm{pH}$ of the endosomes and lysosomes when they decreases to $\mathrm{pH}$ 5-6.

Nanomater sized semiconductor particles can be covalently linked with biorecognition molecules such as peptides, antibodies, nucleic acid, and small molecules ligand as biological labels [52]. The new approach of quantum dots technology with anticancer drug therapy called ZnQ Quantum dots which is loaded with anti cancer agents and encapsulated with biocompatible polymer represent a potential platform to deliver tumor targeted drugs and document the delivery process [53]. The non toxic water dispersed ZnQ quantum dots with long term fluorescence stability can be synthesized by a chemical hydrolysis method encapsulated with chitoson and loaded with anticancer drug. Chitosan enhanced the stability of quantum dots for its hydrophilicity and cationic charge of chitoson [45]. NPs are being developed as delivery vehicles for therapeutic pharmaceuticals such as liposomal NPs (LNPs), encapsulated therapeutic agents for cancer therapy, pegylated form of liposomal encapsulated doxorubicin for breast cancer, layered double hydroxide (LDHs), nanoscale polymer carrier therapy for targeting tumor cells, water soluble polymers drug conjugate to increases half life with potent antitumor effect, 5-flurorouracil loaded iron/ethylcellulose NPs for active targeting of cancer cells can be used in nanomedicine [54-58]. NPs play a vital role in developing new drugs to neural disorders [15]. It is more challenging for delivery of drugs to central nervous system (CNS) and brain but NPs and neuropeptides can over comes these problems and the drug can be delivered in the brain successfully through the carrier such as hexapeptide dalargin, dipep- tide kyotorphin across blood brain barriers (BBB) through endocytosis by endothelical cell lining of the brain blood capillaries. Nimje [59] impart that NPs can be used as carrier such as mannose conjugated solid lipid NPs (SLNPs) that can be exploited for effective and targeted delivery of rifabutin. [60]. The nanosized carriers like micro nanosuspension, liposome, dendrimer, ocular inserts, hydrogels are useful in ocular drug delivery which improves the release profile and reduced toxicity. This method of approach will also increase the efficiency of drug delivery than conventional delivery system. Ladewing [61] reveals that using of layered double hydroxides (LDHs) NPs can be used as carriers for nucleic acids and drug against the general background of bottlenecks that are encountered by cellular delivery system. Nanogels have hydrophilic or amphiphilic polymer chain which can also be used as carriers of drugs and designed spontaneously incorporated biologically active molecules by formation of salt bond, hydrogen bond or hydrophobic interaction [62]. In addition Poly electrolyte nanogels can readily incorporate oppositely changed low molecular mass drug and biomacromolecules such as oligo and polynucleotides (si RNA, DNA) and protein. A general comparison of untargeted and targeted drug delivery by using encapsulated drug system is shown in Figure $\mathbf{1 .}$

\subsection{Role of Nanoparticles as Medicine}

Nanotechnology contributes in management of lung, blood disease and also it counters multiple drug resistance in leukemia by blocking drug efflux from cancer cells and induce efficient delivery of si RNA in to lymphocytes to block apoptosis in sepsis [63]. NPs based thrombocytic agent have potential to improve effectiveness of clot removal and also used in nanodentistry in treatment like dention re-naturalization, permanent hyper sensitivity, complete orthodontic realignments and convalently bonded diamondized enamel [64]. Nanosilver which is a nanoproduct of $100 \mathrm{~nm}$ contains 20-15:000 silver atoms that have strong antibacterial activity which used in wounds and burn healing [65]. Nanocrysalin silver have the property of inhibiting antibiotic resistance and anti septic resistance microbes. Dendrimers is a novel polymers with well defined structure, high molecular uniformity and low polydispersity property that makes them more attractive in development of nanomedicine [66]. Dendrimers based delivery system transports drug across cellular barrier efficiently. Mesoporous silica particle (MSP), layered double hydroxide (LDHS) are used for efficient drug delivery [67,68]. NPs based drug deliveries can target intracellular infection like tuberculosis and also polymeric NPs employing polylactide co-glycolide have more potent anti-tubercular activity [22]. These NPs can be used for site specific delivery 


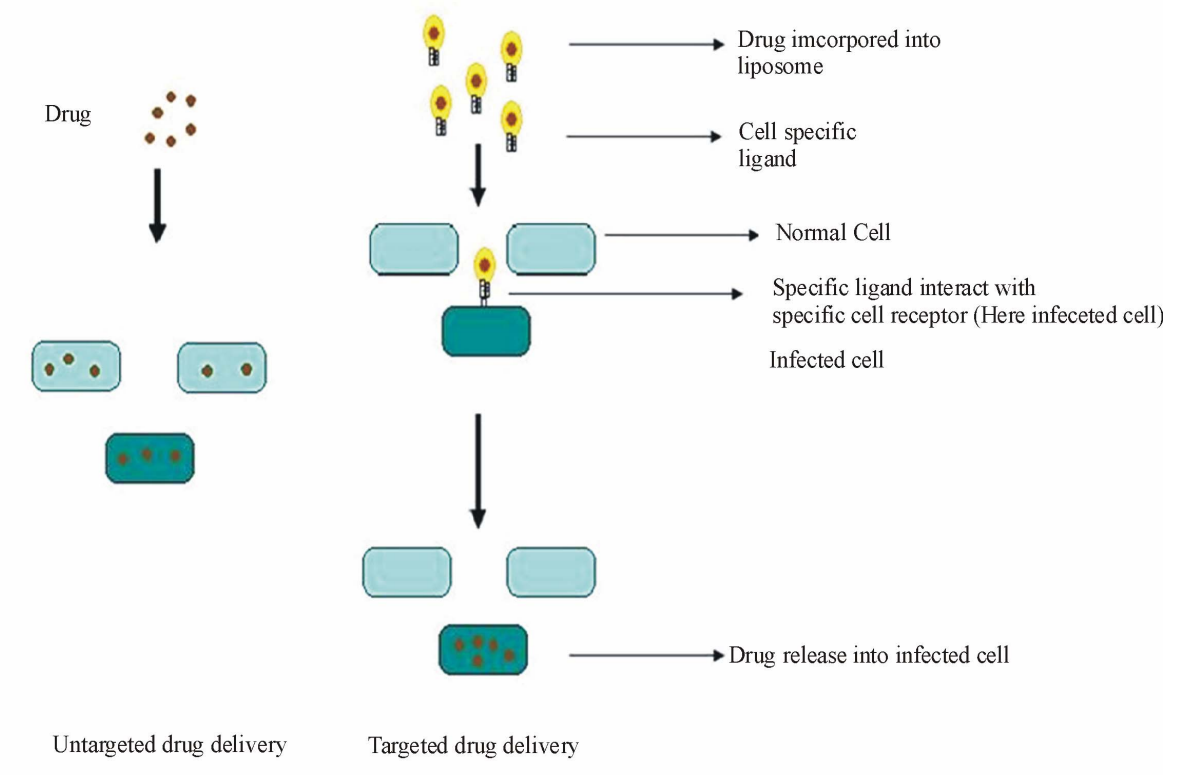

Figure 1. Drug delivery system using nanotechnology.

by avoiding the unwanted toxicity due to non specific distribution and improve the quality of the patients [69]. NPs can act as potent free radical scavenger and they may have anti-inflammatory activity [20]. These NP antioxidants may provide opportunities to counteract the pathogencity of Pseudonomas aeruginosa and its biofilm formation. These advances in nanotechnology research provide new set of research tools, materials, structures and application in nanomedicine in nanotherapy.

\subsection{Nanoparticles Based Diagnostic in Cancer Therapy}

Nanotechnology has provided an advance biomedical research tool in diagnostic imaging, therapy and targeting of NPs to individual cells and sub cellular compartment [70]. The gold NPs in cellular uptake depend upon their size and surface properties which is transported at 300 $500 \mathrm{~nm}$ diameter within the cytoplasm. In advances, NPs is also used in mediating thermal therapy indicating absorption of infrared light, radiofrequency ablation and magnetically induced heating [71]. The use of radio labeled NPs tagged with radio nuclides and fluorescent NPs such as organic dye doped NPs, Quantum dots and multi function NPs which can be conjugated with several functional molecules that may promote new diagnostic tool in cancer therapy [28,72]. Nanotechnology enabled drug delivery approaches usually to reduce the systemic distribution and associated side effects typically observed with conventional chemotherapeutic molecules [73]. Recent research has developed number of NPs such as metal, semiconductor and polymeric particles to be use as imaging probes, diagnosis and as delivery vehicles in cancer therapy [28]. NPs play an important role in cancer diagnosis. The particles such as organic dye, doped polymer, liposomes, and quantum dots are used in cancer diagnosis [74-77]. Multi functional NPs have the capability to simultaneously carry therapeutic agents, squares imaging contrast agent, diamonds and targeting moieties (circle) that can be used as anti cancer agents [28]. Drug loaded NPs can also be used in treating cancer in animal model. Multi functional miscelle has been developed for in vitro cancer cell targeting, distribution imaging and anti cancer delivery [78]. Doxorubicin (DOX) drugs released from micelles have strong effects on the viability of human liver carcinoma cell line (Hep G2). In addition to DOX drugs loaded NPs have greater anti cancer activity in HER -2 over expressing human breast adenocarcinoma cell line (SK-BR-3) [79,80]. Among inorganic nanomaterials, silica or mesoporous silica materials can be used as potential delivery vehicles and imaging probes for their effective biocompatibility and easy surface fictionalization [78,81-85]. NPs play an important role by delivering drug in a targeted manner to the malignant tumor cells by reducing the systemic toxicity of the anti cancer drugs [86]. Rapamycin loaded polymeric (poly (lactide-co-gyycolide) (PLGA) NPs conjugate with antibodies to epidermal growth factor receptor (EGFR) that have efficient and targeted delivery of anticancer drugs.

\subsection{Nanoparticles Mediated Targeting Tumors}

Nanoparticles can deliver anti cancer agent to tumor site by two strategies such as active and passive that offers significant benefits to cancer patients [85,23]. Passive targeting of tumor site will depend upon the nanoparticle 
size and tumor vasculature in order to enhance the efficacy of drugs. The solid tumor increase their surrounding vasculature through angiogenesis in order to grow beyond $1 \mathrm{~mm}-2 \mathrm{~mm}$ diameter but during the development of blood vessels they have several abnormalities like deficiency in pericytes, aberrant basement membrane formation. These abnormalities result in leaky vessels with gap size of $200 \mathrm{~nm}$ to $1.2 \mu \mathrm{m}$ between adjacent endothelial cells that allow extravasations of NPs through these gaps in to extra vascular space [87-89]. These NPs gain access to the tumor that has higher retention times than normal tissues [90]. The leaky vasculature with non effective lymphatic drainage induces the enhanced permeability and retention (EPR) effect lead to accumulation of NPs at tumor site [91-93]. Active targeting of tumor cells by conjugate targeting moieties to NPs lead to accumulation of NPs in tumor sites. The antibodies bind to an antigen on the tumor cell surface and assist NPs drug delivery system to tumor sites [94-97].

Lyp-1 nanoparticles target specific peptide of (PEG -PLGA) NPs to tumor lymph metastasis is a promising carrier to target specific drug delivery to lymphatic metastasis tumor [98]. The nanocarrier allows accumulation of melittin in murine tumor growth without toxicity. In a direct assay the molecularly targeted nanocarriers selectively delivered melittin to multiple tumor targets such as endothelial and cancer cells through a hemifusion mechanism. In animals it may causes regression of precancerous dysplastic lesions and this provides an innovative molecular design for chemotherapy with broad spectrum cytolytic peptides treated for cancer at multiple stages. Silver NPs can act as anti-angiogenic molecules by targeting the activation of P13K/AKT signaling pathway [99]. These silver (Ag) NPs have ability to inhibit angiogenesis, invasiveness, metastasis, vascular endothelial growth factor (VEGF) induced cell proliferation, migration and capillary like tube formation of bovine retinal endothelial cells. In addition silver NPs also have the property of inhibiting the formation of new blood micro vessels induced by VEGF in the mouse matrigel plug assay [99]. In addition NPs mediated targeting of phosphatidylinositol -3-kinase signaling inhibits angiogenesis [100]. NPs enabled targeting the p13K pathway result in inhibition of proliferation and induction of apoptosis of B16F-10 melanoma. Therefore NPs enabled targeting of p13K pathway resulted in inhibition of endothelial cell proliferation and tumor angiogenesis [100]. Singh [101] demonstrated that canine parvovirus NPs (CPVNPs) can be used for targeting tumor cells. The viral particles are nanostructures with nanocontainer for cellular delivery as they have naturally evolved mechanisms for binding to an entering to cells. Canine parvovirus have natural affinity for transferrin receptor (TIRs) canine and human origin and this property could be harnessed as (TIRs) are over expressed by a variety of human tumor cells. CPV based VLPs for TIRs which provides a novel nanomaterial for delivery of a therapy. Labeling nanocrystals with immune cells act as platform technology for nanoimmunotherapy. The combination of plasmid DNA encoding a multimeric soluble form of CD40L (Psp-d-CD40L) can reduced tumor growth which can be established through B16F-10 melanoma tumor [102]. The combination of Toll-like Receptor (TLR) agonists, -C-phosphate-G- (CpG) and poly (i:c) reduces the tumor growth and increase the survival rate. It is also associated with reduction of intra tumoral CD11c+ dendritic cells and an influx of CD8 T cells. The intra tumoral injection of Psp-d-Cb40L containing NPs formed form poly ethylenimine (PEI) in combination with CPG +poly (i:c) may have dramatic anti-tumor effect and it can also treat B16F-10 tumor bearing mice.

\section{Conclusions}

The multidisciplinary field of nanotechnology's application for discovering new molecules and manipulating those available naturally could be excited in its potential to improve health care. Nanotechnology is definitely a medical boon for diagnosis, treatment and prevention of various diseases including cancer. It supports and expands the scientific advances in genomic and proteomics and builds on our understanding of the molecular underpinnings of cancer and its treatment. We then review the current state of the art of nanoparticle-based therapeutics that have reached the clinic for its efficient advantage as drug carrier which are high stability, high carrier capacity, feasibility of incorporation of both hydrophilic and hydrophobic substances of variable routes of administration including oral application and inhalation. Multi functional NPs also have the capability to concurrently carry therapeutic agents, squares imaging contrast agent, diamonds and targeting moieties which can be used as anti-cancer agents. Interestingly pharmaceutical sciences are using NPs to reduce toxicity and side effects of drugs. The kind of hazards that are introduced by using NPs for drug delivery are beyond that posed by conventional hazards imposed by chemicals in classical delivery matrices. Predicting the future of nanotechnology in drug delivery system is not simple due to its fast developing technology and changing rapidly. Additional research is required in multifunctional NPs based drug delivery systems to overcome the problems for effective therapy without side effects which can improve the quality of life in cancer patients.

\section{Acknowledgements}

The valuable help and support of Dr. Patrick Gomez, 
Director, School of Biotechnology and Health Sciences, Karunya University is gratefully acknowledged.

\section{REFERENCES}

[1] S. Majuru and O. Oyewumi, "Nanotechnology in Drug Development and Life Cycle Management," Nanotechnology in Drug Delivery, Vol. 10, No. 4, 2009, pp. 597-619. doi:10.1007/978-0-387-77668-2_20

[2] K. K. Jain, "The Role of Nanobiotechnology in Drug Discovery,” Drug Discover Today, Vol. 10, No. 21, 2005, pp. 1435-1442. doi:10.1016/S1359-6446(05)03573-7

[3] C. Buzea, I. I. Pacheco and K. Robbie, "Nanomaterials and Nanoparticles: Sources and Toxicity," Biointerphases, Vol. 2, No. 4, 2007, pp. 17-71.

[4] B. D. Fahlman, "Materials Chemistry," Springer, Berlin, 2007, pp. 282-283. doi:10.1007/978-1-4020-6120-2

[5] W. Yang, J. I. Peters, R. O. and Williams, Eds., "Inhaled Nanoparticles - A Current Review," International Journal of Pharmceutical, Vol. 22, No. 356, 2008, pp. 239-247. doi:10.1016/j.ijpharm.2008.02.011

[6] B. Mishra, B. B Patel and S. Tiwari, "Colloidal Nanocarriers: A Review on Formulation Technology, Types and Applications toward Targeted Drug Delivery," Nanomedicine, Vol. 6, No. 1, 2010, pp. 9-24. doi:10.1016/j.nano.2009.04.008

[7] Z. Ghalanbor, S. A. Marashi and B. Ranjbar, "Nanotechnology Helps Medicine: Nanoscale Swimmers and Their Future Applications,” Medical Hypotheses, Vol. 65, No. 1, 2005, pp. 198-199. doi:10.1016/j.mehy.2005.01.023

[8] T. Kubik, K. Bogunia-Kubik and M. Sugisaka, "Nanotechnology on Duty in Medical Applications," Current Pharmaceutical Biotechnology, Vol. 6, No. 1, 2005, pp. 17-33.

[9] S. N. Kundra, "Toward the Emergence of Nanoneurosurgery: Part III-Nanomedicine: Targeted Nanotherapy, Nanosurgery and Progress toward the Realization of Nanoneurosurgery,” Neurosurgery, Vol. 62, No. 6, 2008, p. 1384. doi:10.1227/01.neu.0000333332.65576.c7

[10] A. Cavalcanti, B. Shirinzadeh and R. A. Freitas, "Medical Nanorobot Architecture Based on Nanobioelectronics," Recent Patents on Nanotechnology, Vol. 1 No. 1, 2007, pp. 1-10. doi:10.2174/187221007779814745

[11] M. Boukallel, M. Gauthier, M. Dauge, E. Piat and J. Abadie, "Smart Microrobots for Mechanical Cell Characterization and Cell Convoying," IEE Transaction on Biomedical Engneering, Vol. 54, No. 8, 2007, pp. 1536-1540. doi:10.1109/TBME.2007.891171

[12] D. A. La Van, T. McGuire and R. Langer, "Small-Scale Systems for in vivo Drug Delivery,” Nature Biotechnology, Vol. 21, No. 10, 2003, pp. 1184-1191. doi:10.1038/nbt876

[13] A. Cavalcanti and B. Shirinzadeh, "Nanorobot Architecture for Medical Target Identification,” Nanotechnology, Vol. 19, 2008, p. 015103. doi:10.1088/0957-4484/19/01/015103

[14] R. R. Zhu, L. L. Qin, M. Wang, S. L. Wang, R. Zhang, Z.
X. Liu, X. Y. Sun and S. D. Yao, "Preparation, Characterization, and Anti-tumor Property of PodophyllotoxinLoaded Solid Lipid Nanoparticles,” Nanotechnology, Vol. 20, No. 5, 2009, p. 55702. doi:10.1088/0957-4484/20/5/055702

[15] K. K. Jain, "Role of Nanotechnology in Development of New Therapies for Diseases of the Nervous System," Nanomedicine, Vol. 1, No. 1, 2006, pp. 9-12. doi:10.2217/17435889.1.1.9

[16] J. C. Pickup, Z. L. Zhi, F. Khan, T. Saxl and D. J. Birch, "Nanomedicine and Its Potential in Diabetes Research and Practice,” Diabetes/Metabolism Research and Reviews, Vol. 24, No. 8, 2008, pp. 604-610. doi:10.1002/dmrr.893

[17] L. Jianguo, Z. Li, Z. Yi, W. Huanan, L. Jidong, Z. Qin and L. Yubao, "Development of Nanohydroxyapatite/ Polycarbonate Composite for Bone Repair," Journal of Biomaterials Applications, Vol. 24, No. 1, 2009, pp. 31-45. doi:10.1177/0885328209102756

[18] A. Nowacek, L. M. Kosloski and H. E. Gendelman, "Neurodegenerative Disorders and Nanoformulated Drug Development," Nanomedicine, Vol. 4, No. 5, 2009, pp. 541-555. doi:10.2217/nnm.09.37

[19] D. N. Patel and S. R. Bailey, "Nanotechnology in Cardiovascular Medicine," Catheterization and Cardiovascular Interventions, Vol. 69, No. 5, 2007, pp. 643-654. doi:10.1002/ccd.21060

[20] S. F. Elswaifi, J. R. Palmieri, K. S. Hockey and B. A. Rzigalinski, "Antioxidant Nanoparticles for Control of Infectious Diseases,” Infect Disorder Drug Target, Vol. 9, No. 4, 2009, pp. 445-452.

[21] M. Smola, T. Vandamme and A. Sokolowski, "Nanocarriers as Pulmonary Drug Delivery Systems to Treat and to Diagnose Respiratory and Non Respiratory Diseases," International Journal of Nanomedicine, Vol. 3, No. 1, 2008, pp. 1-19.

[22] R. Pandey and G. K. Khuller, "Nanotechnology Based Drug Delivery System(s) for the Management of Tuberculosis,” Indian Journal of Experimental Biology, Vol. 44, No. 5, 2006, pp. 357-366.

[23] Y. Y. Liu, H. Miyoshi and M. Nakamura, "Nanomedicine for Drug Delivery and Imaging: A Promising Avenue for Cancer Therapy and Diagnosis Using Targeted Functional Nanoparticles," International Journal of Cancer, Vol. 120, No. 12, 2007, pp. 2527-2537. doi:10.1002/ijc.22709

[24] X. Wang, L. L. Yang, Z. Chen and D. M. Shin, “Application of Nanotechnology in Cancer Therapy and Imaging," A Cancer Journal for Clinicians, Vol. 58, 2008, pp. 97-110. doi:10.3322/CA.2007.0003

[25] K. Riehemann, S. W. Schneider, T. A. Luger, B. Godin, M. Ferrari and H. Fuchs, "Nanomedicine-Challenge and Perspectives," Angewandte Chemie International Edition, Vol. 48. No. 5, 2009, pp. 872-897. doi:10.1002/anie.200802585

[26] R. Sinha, G. J. Kim, S. Nie and D. M. Shin, "Nanotechnology in Cancer Therapeutics: Bioconjugated Nanoparticles for Drug Delivery," Molecular Cancer Therapeutics, Vol. 5, No. 8, 2006, pp. 1909-1917. doi:10.1158/1535-7163.MCT-06-0141 
[27] K. J. Cho, X. Wang, S. M. Nie and D. H. Shin, "Therapeutic Nanoparticles for Drug Delivery in Cancer," Clinical Cancer Research, Vol. 14, 2008, pp. 1310-1316. doi:10.1158/1078-0432.CCR-07-1441

[28] S. Jiang, M. K. Ganesammandhan and Y. Zhang, “Optical Imaging Guided Cancer Therapy with Fluorescent Nanoparticles," Journal of the Royal Society Interface, Vol. 7, No. 42, 2010, pp. 3-18. doi:10.1098/rsif.2009.0243

[29] D. Castanotto and J. J. Rossi, "The Promises and Pitfalls of RNA-Interference-Based Therapeutics,” Nature, Vol. 457, No. 7228, 2009, pp. 426-433.

[30] S. Sandhiya, S. A. Dkhar and A. Surendiran, "Emerging Trends of Nanomedicine-An Overview," Fundamental \& Clinical Pharmacology, Vol. 23, No. 3, 2009, pp. 263-269. doi:10.1111/j.1472-8206.2009.00692.x

[31] R. D. Hofheinz, S. U. Gnad-Vogt, U. Beyer and A. Hochhaus, "Liposomal Encapsulated Anti-cancer Drug," Anticancer Drugs, Vol. 16, No. 7, 2005, pp. 691-707. doi:10.1097/01.cad.0000167902.53039.5a

[32] S. M. Moghimi and J. Szebeni, "Stealth Liposomes and Long Circulating Nanoparticles: Critical Issues in Pharmacokinetics, Opsonization and Protein-Binding Properties,” Progress in Lipid Research, Vol. 42, No. 6, 2003, pp. 463-478. doi:10.1016/S0163-7827(03)00033-X

[33] D. K. Sarker, "Engineering of Nanoemulsions for Drug Delivery,” Current Drug Delivery, Vol. 2, 2005, pp. 297-310. doi:10.2174/156720105774370267

[34] S. A. Agnihotri, N. N. Mallikarjuna and T. M. Aminabhavi, "Recent Advances on Chitosan-Based Micro- and Nanoparticles in Drug Delivery," Journal of Controlled Release, Vol. 100, No. 1, 2004, pp. 5-28. doi:10.1016/j.jconrel.2004.08.010

[35] L. J. Lee, "Polymer Nano-Engineering for Biomedical Applications," Annals of Biomedical Engineering, Vol. 34, No. 1, 2006, pp. 75-88. doi:10.1007/s10439-005-9011-6

[36] A. K. Cherian, A. C. Rana and S. K. Jain, "Self-Assembled Carbohydratestabilized Ceramic Nano-particles for the Parenteral Delivery of Insulin,” Drug Development and Industrial Pharmacy, Vol. 26, No. 4, 2000, pp. 459-463. doi:10.1081/DDC-100101255

[37] L. R. Hirsch, A. M. Gobin, A. R. Lowery, F. Tam, R. A. Drezek, N. J. Halas and J. L. West, "Metal Nanoshells," Annals of Biomedical Engineering, Vol. 34, No. 1, 2006, pp. 15-22. doi.org/10.1007/s10439-005-9001-8

[38] S. Bosi, T. Da Ros, G. Spalluto and M. Prato, "Fullerene Derivatives: An Attractive Tool for Biological Applications," European Journal of Medicinal Chemistry, Vol. 38, No. 11-12, 2003, pp. 913-923. doi:10.1016/j.ejmech.2003.09.005

[39] G. Pagona and N. Tagmatarchis, "Carbon Nanotubes: Materials for Medicinal Chemistry and Biotechnological Applications," European Journal of Medicinal Chemistry, Vol. 13, 2006, pp. 1789-1798. doi:10.2174/092986706777452524

[40] J. Weng and J. Ren, "Luminescent Quantum Dots: A Very Attractive and Promising Tool in Biomedicine,"
Current Medicinal Chemistry, Vol. 13, 2006, pp. 97-909. doi:10.2174/092986706776361076

[41] M. E. Davis, Z. G. Chen and D. M. Shin, "Nanoparticle Therapeutics: An Emerging Treatmentmodality for Cancer,” Nature Reviews Drug Discovery, Vol. 7, 2008, pp. 771-782. doi:10.1038/nrd2614

[42] H. Pelicano, D. S. Martin, R. H, Xu and P. Huang, "Glycolysis Inhibition for Anticancer Treatment," Oncogene, Vol. 25, 2006, pp. 4633-4646. doi:10.1038/sj.onc.1209597

[43] S. S. Nath, D. Chakdar, G. Gope and D. K. Avasthi, "Characterizations of CdS and ZnS Quantum Dots Prepared by Chemical Method on SBR Latex,” AZojono Journal of Nanotechnology Online, 2008. doi:10.2008, 2240.

[44] V. R. Reddy, "Gold Nanoparticles: Synthesis and Applications,” Thieme eJournals, Vol. 2006, No. 11, 2006, pp. 1791-1792.

[45] R. Das, S. S. Nath, D. Chakdar, G. Gope and R. Bhattacharjee, "Preparation of Silver Nanoparticles and Their Characterization," AZojono Journal of Nanotechnology Online, Vol. 5, No. 10, 2009, p. 2240.

[46] X. Wang, J. Zhuang, Q. Peng and Y. Li, "General Strategy for Nanocrystal Synthesis,” Nature, Vol. 437, No. 7055, 2005, pp. 121-124. doi:10.1038/nature03968

[47] H. Zhu, C. Zhang and Y. Yin, "Novel Synthesis of Copper Nanoparticles: Influence of the Synthesis Conditions on the Particle Size,” Nanotechnology, Vol. 16, No. 12, 2005, p. 3079. doi:10.1088/0957-4484/16/12/059

[48] Y. Wei, H. Xie, L. Chen, Y. Li and C. Zhang, "Controlled Synthesis of Narrow-Dispersed Copper Nanoparticles," Journal of Dispersion Science and Technology, Vol. 31, No. 3, 2010, pp. 364-367. doi:10.1080/01932690903196193

[49] L. S. Jackson and K. Lee, "Microencapsulation and the Food Industry,” Lebensmittel—Wissenschaft Technologie, 1991.

[50] S. Gelperina, K. Kisich, M. D. Iseman and L. Heifets, "The Potential Advantages of Nanoparticle Drug Delivery Systems in Chemotherapy of Tuberculosis," American Journal of Respiratory and Critical Care Medicine, Vol. 172, No. 12, 2005, pp. 1487-1490. doi:10.1164/rccm.200504-613PP

[51] Y. Bae, N. Nishiyama, S. Fukushima, H. Koyama, M. Yasuhiro and K. Kataoka, "Preparation and Biological Characterization of Polymeric Micelle Drug Carriers with Intracellular pH-Triggered Drug Release Property: Tumor Permeability, Controlled Subcellular Drug Distribution, and Enhanced in vivo Antitumor Efficacy,” Bioconjugate Chemistry, Vol. 16, No. 1, 2005, pp. 122-130. doi:10.1021/bc0498166

[52] E. Physica, "Quantum Dots in Biology and Medicine,” Low-Dimensional Systems and Nanostructures, Vol. 25, No. 1, 2004, pp. 1-12. doi:10.1016/j.physe.2004.07.013

[53] Q. Yuan, S. Hein and R. D. Misra, "New Generation of Chitosan-Encapsulated ZnO Quantum Dots Loaded with Drug: Synthesis, Characterization and in vitro Drug De- 
livery Response,” Acta Biomaterialia, Vol. 6, No. 7, 2010, pp. 2732-2739. doi:10.1016/j.actbio.2010.01.025

[54] D. B. Fenske, A. Chonn and P. R. Cullis, "Liposomal Nanomedicines: An Emerging Field," Toxicology $\mathrm{Pa}$ thology, Vol. 36, No. 1, 2008, pp. 21-29. doi:10.1177/0192623307310960

[55] S. Praveen and S. K. Sahoo, "Polylymeric Nanoparticles for Cancer Therapy,” Journal of Drug Targeting, Vol. 16, No. 2, 2008, pp. 108-123. doi:10.1080/10611860701794353

[56] Y. Luo and G. D. Prestwich, "Cancer Targeted Polymeri Drug,” Current Cancer Drug Targets, Vol. 2, No. 3, 2002, pp. 209-226. doi:10.2174/1568009023333836

[57] J. L Arias, M. López-Viota, A. V. Delgado and M. A. Ruiz, "5-Fluorouracil-Loaded Iron/Ethylcellulose (Core/ Shell) Nanoparticles for Active Targeting of Cancer," Journal of Drug Targeting, Vol. 17, No. 10, 2009, p. 813. doi:10.3109/10611860903244207

[58] T. Tanaka, P. Decuzzi , M. Cristofanilli, J. H. Sakamoto, E. Tasciotti, F. M. Robertson and M. Ferrari, "Nanotechnology for Breast Cancer Therapy,” Biomedical Microdevices, Vol. 11, No. 1, 2009, pp. 49-63. doi:10.1007/s10544-008-9209-0

[59] N. Nimje, A. Agarwal, G. K. Saraogi, N. Lariya, G. Rai, H. Agrawal and G. P. Agrawal, "Nanoparticulate Carriers of Rifabutin for Alveolar Targeting,” Journal of Drug Targeting, Vol. 17, No. 10, 2009. pp. 777-787. doi:10.3109/10611860903115308

[60] S. Wadhwa, R. Paliwal, S. R. Paliwal and S. P. Vyas, "Nanocarriers in Ocular Drug Delivery," Current Pharmceutical Design, Vol. 15, No. 23, 2009, pp. 2724-2750. doi:10.2174/138161209788923886

[61] K. Ladewing, Z. P. Xu and G. C. Lu, "Layered Double Hydroxide Nanoparticle in Gene and Drug Delivery," Expert Opinion on Drug Delivery, Vol. 6, No. 9, 2009, pp. 907-922. doi:10.1517/17425240903130585

[62] A. V. Kabanov and S. K. Vinogradov, "Nanogels as Pharmaceutical Carriers: Finite Network of Infinite Capabilities," Angewandte Chemie International Edition, Vol. 48, No. 30, 2009, pp. 5418-5429. doi:10.1002/anie.200900441

[63] D. B. Buxton, "Nanomedicine for the Management of Lung and Blood Diseases," Nanomedicine, Vol. 4, No. 3. 2009, pp. 331-339. doi:10.2217/nnm.09.8

[64] R. A. Freitas, “Nanodentistry,” Journal of American Dental Association, Vol. 131, No. 11, 2000, pp. 1559-1565.

[65] J. B. Lyczak and P. J. Schechter, "Nanocrystalline Silver Inhibits Antibiotic, Antiseptic-Resistant Bacteria," Clinical Pharmacology Therapeutics, Vol. 77, 2005, p. 60. doi:10.1016/j.clpt.2004.12.119

[66] A. Samad, M. I. Alam and K. Saxena, "Dendrimers: A Class Of Polymers in the Nanotechnology for the Delivery of Active Pharmaceuticals," Current Pharmceutical Design, Vol. 15, No. 25, 2009, pp. 2958-2969. doi:10.2174/138161209789058200

[67] L. Pasqua, S. Cundari, C. Ceresa and G. Cavaletti, "Recent Development, Applications, and Perspectives of
Mesoporous Silica Particles in Medicine and Biotechnology,” Current Medical Chemistry, Vol. 16, No. 23, 2009, pp. 3054-3063. doi:10.2174/092986709788803079

[68] K. Ladewig, Z. P. Xu and G. C. Lu, "Layered Double Hydroxide Nanoparticles in Gene and Drug Delivery," Expert Opinion on Drug Delivery, Vol. 6, No. 9. 2009, pp. 907-922. doi:10.1517/17425240903130585

[69] H. Devalapally, A. Chakilam and M. M. Amiji, "Role of Nanothechnology in Pharmaceutical Product Development," Journal of Pharmaceutical Sciences, Vol. 96, No. 10, 2007, pp. 2547-2567. doi:10.1002/jps.20875

[70] B. D. Chithrani, J. Stewart, C. Allen and D. A. Jaffray, "Intracellullar Uptake, Transport, and Processing of Nanostructures in Cancer Cells,” Nanomedicine, Vol. 5, No. 2, 2009, pp. 118-127. doi:10.1016/j.nano.2009.01.008

[71] E. S. Day, J. G. Morton and J. L. West, "Nanoparticles for Thermal Cancer Therapy," Journal of Biomechanical Engneering, Vol. 131, No. 7, 2009, p. 74001. doi:10.1115/1.3156800

[72] G. Ting, C. H. Chang and H. E. Wang, "Cancer Nanotargeted Radiopharmaceutical for Tumor Imaging and Therapy,” Anticancer Research, Vol. 29, No. 10, 2009, pp. 4107-4108.

[73] B. Thierry, "Drug Nanocarriers and Functional Nanoparticles Application in Cancer Therapy," Current Drug Delivery, Vol. 6, No. 4, 2009, pp. 391-403. doi:10.2174/156720109789000474

[74] S. Santra, D. Dutta, G. A.Walter and B. M. Moudgil, "Fluorescent Nanoparticle Probes for Cancer Imaging," Technology in Cancer Research and Treatment, Vol. 4, No. 6, 2005, pp. 593-602.

[75] K. Licha and C. Olbrich, "Optical Imaging in Drug Discovery and Diagnostic Applications,” Advanced Drug Delivery Reviews, Vol. 57, No. 8, 2005, pp. 1087-1108. doi:10.1016/j.addr.2005.01.021

[76] P. Grodzinski, M. Silver and L. K. Molnar, "Nano-Technology for Cancer Diagnostics: Promises and Challenges," Expert Review of Molecular Diagnostics, Vol. 6. No. 3, 2006, pp. 307-318. doi:10.1586/14737159.6.3.307

[77] J. Rao, A. Dragulescu-Andrasi and H. Yao, "Fluorescence Imaging in vivo: Recent Advances," Current Opinion in Biotechnology, Vol. 18, No. 1, 2007, pp. 17-25. doi:10.1016/j.copbio.2007.01.003

[78] D. M. Huang, Y. Hung, B. S. Ko, S. C. Hsu, W. H. Chen, C. L. Chien, C. P. Tsai, C. T. Kuo, J. C. Kang, C. S. Yang, C. Y. Mou and Y. C. Chen, "Highly Efficient Cellular Labeling of Mesoporous Nanoparticles in Human Mesenchymal Stem Cells: Implication for Stem Cell Tracking," Journal of the Federation of American Societies for Experimental Biology, Vol. 19, No. 14, 2005, pp. 2014-2016.

[79] J. Kim, J. E. Lee, S. H. Lee, J. H. Yu, J. H. Lee, T. G. Park and T. Hyecon. "Designed Fabrication of a Multifunctional Polymer Nanomedical Platform for Simultaneous Cancer-Targeted Imaging and Magnetically Guided Drug Delivery,” Advanced Materials, Vol. 20, 2008, pp. 478-483. doi:10.1002/adma.200701726 
[80] K. Shah, A. Jacobs, X. O. Breakefield and R. Weissleder, "Molecular Imaging of Gene Therapy for Cancer," Gene Therapy, Vol. 11, No. 15, pp. 1175-1187. doi:10.1038/sj.gt.3302278

[81] Y. S. Lin, S. H. Wu, Y. Hung, Y. H. Chou, C. Chang, M. L. Lin, C. P. Tsai and C. Y. Mou, "Multifunctional Composite Nanoparticles: Magnetic, Luminescent, and Mesoporous," Chemistry of Materials, Vol. 18, No. 22, 2006, pp. 5170-5172. doi:10.1021/cm061976z

[82] S. T. Selvan, P. K. Patra, C. Y. Ang and J. Y. Ying, "Synthesis of Silica-Coated Semiconductor and Magnetic Quantum Dots and Their Use in the Imaging of Live Cells," Angewandte Chemie International Edition, Vol. 46, No. 14, 2007, pp. 2448-2452. doi:10.1002/anie.200604245

[83] S. H. Wu, Y. S. Lin, Y. Hung, Y. H. Chou, Y. H. Hsu, C. Chang and C. Y. Mou, "Multifunctional Mesoporous Silica Nanoparticles for Intracellular Labeling and Animal Magnetic Resonance Imaging Studies,” Chembiochem, Vol. 9, No. 1, 2008, pp. 53-57. doi:10.1002/cbic.200700509

[84] C. H. Lee, S. H. Cheng, Y. J. Wang, Y. C. Chen, N. T. Chen, J. Souris, C. T. Chen, C. Y. Mou, C. H. Yang and L. W. Lo, "Near-Infrared Mesoporous Silica Nanoparticles for Optical Imaging: Characterization and in vivo Biodistribution," Advanced Functional Materials, Vol. 19, No. 2, 2009, pp. 215-222. doi:10.1002/adfm.200800753

[85] J. D. Byrne, T. Betancourt and L. Brannon-Peppas, “Active Targeting Schemes for Nanoparticle Systems in cancer Therapeutics,” Advanced Drug Delivery Reviews, Vol. 60, No. 15, 2008, pp. 1615-1626. doi:10.1016/j.addr.2008.08.005

[86] S. Acharya, F. Dilnawaz and S. K. Sahoo, "Targeted Epidermal Growth Factor Receptor Nanoparticle Bioconjugates for Breast Cancer Therapy,” Biomaterials, Vol. 30, No. 29, 2009, pp. 5737-5750.

doi:10.1016/j.biomaterials.2009.07.008

[87] J. Folkman, "What Is the Evidence that Tumors are Angiogenesis Dependent?” Journal of naternational Cancer Insititution, Vol. 82, No. 1, 1990, pp. 4-6. doi:10.1093/jnci/82.1.4

[88] J. Folkman and Y. Shing, “Angiogenesis,” Journal of Biology Chemistry, Vol. 267, No. 10, 1992, pp. 931-934.

[89] D. F. Baban and L. W. Seymour, "Control of Tumour Vascular Permeability," Advanced Drug Delivery Reviews, Vol. 34, No. 1, 1998, pp. 109-119. doi:10.1016/S0169-409X(98)00003-9

[90] J. W. Baish, Y. Gazit, D. A. Berk, M. Nozue, L. T. Baxter and R. K. Jain, "Role of Tumor Vascular Architecture in Nutrient and Drug Delivery: An Invasion Percolation-Based Network Model,” Microvascular Research, Vol. 51, No. 3, 1996, pp. 327-346. doi:10.1006/mvre.1996.0031

[91] R. K. Jain, "Delivery of Molecular Medicine to Solid Tumors: Lessons from in vivo Imaging of Gene Expression and Function," Journal of Controlled Release, Vol. 74,
No. $1-3,2001$, pp. 7-25. doi:10.1016/S0168-3659(01)00306-6

[92] R. Duncan, "The Dawning Era of Polymer Therapeutics," Nature Reviews Drug Discovery, Vol. 2, No. 5, 2003, pp. 347-360. doi:10.1038/nrd1088

[93] L. Brannon-Peppas and J. O. Blanchette, "Nanoparticle and Targeted Systems for Cancer Therapy,” Advanced Drug Delivery Reviews, Vol. 56, No. 11, 2004, pp. 1649-1659. doi:10.1016/j.addr.2004.02.014

[94] K. Ulbrich, T. Etrych, P. Chytil, M. Jelínková and B. Ríhová, "Antibody-Targeted Polymer-Doxorubicin Conjugates with $\mathrm{pH}$-Controlled Activation,” Journal of Drug Targeting, Vol. 12, No. 8, 2004, pp. 477-489. doi:10.1080/10611860400011869

[95] Z. Xu, W. Gu, J. Huang, H. Sui, Z. Zhou, Y. Yang, Z, Yan and Y. I. Li, "In vitro and in vivo Evaluation of Actively Targetable Nanoparticles for Paclitaxel Delivery," International Journal of Pharmaceutics, Vol. 288, No. 2, 2005, pp. 361-368. doi:10.1016/j.ijpharm.2004.10.009

[96] J. Cheng, B. A. Teply, I. Sherifi, J. Sung, G. Luther, F. X. Gu, E. Levy-Nissenbaum, A. F. Radovic-Moreno, R. Langer and O. C. Farokhzad, "Formulation of Functionalized PLGA-PEG Nanoparticles for in vivo Targeted Drug Delivery,” Biomaterials, Vol. 28, No. 5, 2007, pp. 869-876. doi:10.1016/j.biomaterials.2006.09.047

[97] S. Díez, G. Navarro and C. T. de ILarduya, "In vivo Targeted Gene Delivery by Cationic Nanoparticles for Treatment of Hepatocellular Carcinoma," The Journal of Gene Medicine, Vol. 11, No. 1, 2009, pp. 38-45. doi:10.1002/jgm.1273

[98] G. Luo, X. Yu, C. Jin, F. Yang, D. Fu, J. Long, J. Xu, C. Zhan and W. Lu, "Lyp-1 Conjugated Nanoparticles for Targeting Drug Delivery to Lymphatic Metastatic Tumours," International Journal of Pharmaceutics, Vol. 385, No.1-2, 2010, pp. 150-156. doi:10.1016/j.ijpharm.2009.10.014

[99] S. Gurunathan, K. J. Lee, K. Kalishwaralal, S. Sheikpranbabu, R. Vaidyanathan and S. H. Eom, "Antiangiogenic Properties of Silver Nanoparticles,” Biomaterials, Vol. 30, No. 31, 2009, pp. 6341-6350. doi:10.1016/j.biomaterials.2009.08.008

[100] R. Harfouche, S. Basu, S. Soni, D. M. Hentschel, R. A. Mashelkar and S. Sengupta, "Nanoparticles Mediated Targeting of Phosphatidylinositol-3-Kinase Signaling Inhibits Angiogenesis,” Angiogenesis, Vol. 12, No. 4, 2009, pp. 325-338. doi:10.1007/s10456-009-9154-4

[101] P. Singh, “Tumour Targeting Using Canine Parvovirus Nanoparticles," Current Topics in Microbiology and Immunology, Vol. 327, 2009, pp. 123-141.

[102] G.W. Stone, S. Barzee, V. Snarsky, C. Santucci, B. Tran, R. Langer, G. T. Zugates, D. G. Anderson and R. S. Kornbluth, "Nanoparticle Delivered Multimeric Soluble CD40 LDNA Combined with Like Receptor Agonists as a Treatment for Melanoma,” PLOS One, Vol. 4, No. 10, 2009, p. 7334. doi:10.1371/journal.pone.0007334 